\title{
Achievement of the ITER NBI Ion Source Parameters for Hydrogen at the Test Facility ELISE and Present Status for Deuterium
}

\author{
U. Fantz, D. Wünderlich, R. Riedl, B. Heinemann, F. Bonomo and the NNBI-Team \\ Max-Planck-Institut für Plasmaphysik, Boltzmannstr. 2, 85748 Garching, Germany
}

\begin{abstract}
The ELISE test facility, being in operation since 2013, with its half-size ITER ion source accompanies the ITER NBI development program. Besides consolidation of the ion source design, solving technical problems that arise during operation, gaining early experience in ion source operation and maintenance, ELISE has the task to demonstrate the required ion source parameters required by ITER. ELISE made remarkable progress since then, starting from short pulses $\left(10 \mathrm{~s}\right.$ ) to the ITER target of $1000 \mathrm{~s}^{-}$for $\mathrm{H}^{-}$and $3600 \mathrm{~s}$ for $\mathrm{D}^{-}$(with the constraint to extract the beam repetitively for $10 \mathrm{~s}$ every $\approx 150 \mathrm{~s}$ ). For hydrogen, above $90 \%$ of the required level for the extracted current density has been achieved being limited by the available high voltage power supply and the RF power. For deuterium the performance is limited by the much larger amount of co-extracted electrons; here $65 \%$ of the extracted current density is demonstrated for the duration of almost one hour. One of the challenges is the strong temporal dynamics of the co-extracted electrons and several measures were taken as the introduction of potential rods, modifications of the magnetic filter field configuration and improved caesium conditioning. Testing the magnetic field configuration planned for the test facility MITICA (prototype of the ITER beamline) showed very good performance but indicated also that the field strength should be kept variable to control ions and electrons for the two isotopes individually. Regarding the beam homogeneity the global uniformity of better than $90 \%$ is usually achieved. Measurements of the beam divergence by beam emission spectroscopy revealed higher divergences for the horizontal lines-of-sight than for the vertical ones due to the non-compensated zig-zag deflection of the beamlets by the magnets in the extraction grid.
\end{abstract}

Keywords: Negative Ion Source, Neutral Beam Injection, ITER NBI, ELISE.

\section{Introduction}

The two neutral beam injection (NBI) beamlines for heating and current drive at ITER are based on generation and acceleration of negative ions and are designed to inject up to $16.5 \mathrm{MW}$ per beamline of either $870 \mathrm{keV} \mathrm{H}$ atoms for $1000 \mathrm{~s}$ or $1 \mathrm{MeV} \mathrm{D}$ atoms up to one hour [1,2]. Due to limited neutralization efficiency and losses in the beamline the required accelerated ion currents are $46 \mathrm{~A}$ for $\mathrm{H}^{-}$and $40 \mathrm{~A}$ for $\mathrm{D}^{-}$. Taking into account $30 \%$ stripping losses of negative ions in the 7-stage accelerator system, the ion source has to deliver extracted current densities of $329 \mathrm{~A} / \mathrm{m}^{2}$ for $\mathrm{H}^{-}$and $286 \mathrm{~A} / \mathrm{m}^{2}$ for $\mathrm{D}^{-}$at a filling pressure of $0.3 \mathrm{~Pa}$, extracted from 1280 apertures with a diameter of $14 \mathrm{~mm}$ each. The co-extraction of electrons is unavoidable and these are deflected by magnets embedded in the second grid, the extraction grid (EG). The electrons are impinging on the front surface of this grid and cause high heat loads on localized spots (up to $40 \mathrm{MW} / \mathrm{m}^{2}$ [3]). Being already at the limit of engineering capabilities of this component, the co-extracted electrons should be less (max. equal) than the nominally extracted negative ions. The core divergence of the beamlets is expected to be below $7 \mathrm{mrad}$ and the beam uniformity should be better than $90 \%$ to avoid beam scraping at beamline components.

The ELISE test facility is an integral part of the roadmap towards reliable operation of ITER NBI with focus on the ion source, offering also a high experimental flexibility [2,4]. ELISE went into operation in 2013 with the goal to demonstrate at an ion source of half the ITER- size $\left(1 \times 1 \mathrm{~m}^{2}\right.$ source area with 640 apertures for extraction and acceleration) the ITER ion source requirements for hydrogen and deuterium and to contribute to the overall program towards reliable ITER NBI operation. This comprises the early consolidation of the ion source design based on the prototype of the modular RF-driven concept $[5,6]$, early experience with operation and maintenance of the beam source and accompanying the program of the Neutral Beam Test Facilities (NBTF hosted by Consorzio RFX, Padua, [7]).

\section{ELISE test facility}

The ELISE test facility comprises an RF driven ion source using four drivers for plasma generation and one expansion chamber representing half the height but the full width of the ITER source, an extraction and acceleration system and a tank which accommodates cryo pumps and the diagnostic calorimeter (see Ref. [8] and references therein for more details).

\subsection{Ion source}

The plasma illuminates an area of about $1 \times 1 \mathrm{~m}^{2}$, accommodating the grid system, as sketched in the vertical cut shown in Fig. 1. Unlike at ITER, the source vessel is in air allowing easy source access and modifications, but the four drivers are enclosed in a vacuum containment, called dome, such that the RF drivers are operated in vacuum like at ITER. Each horizontal pair of RF drivers is fed in series by one RF 
generator (1 MHz frequency). RF powers of up to $180 \mathrm{~kW}$ were possible using the tube-based oscillators with which operation was started [6]. Later on, solid-state RF generators with $150 \mathrm{~kW}$ maximum power became available providing easier matching and increasing the reliability of the RF system [9]. According to the present planning, the ITER sources will be equipped with four tube-based RF generators rated to $200 \mathrm{~kW}$ power each, connected to two drivers in series, thus having available a power of $100 \mathrm{~kW} /$ driver for plasma generation.

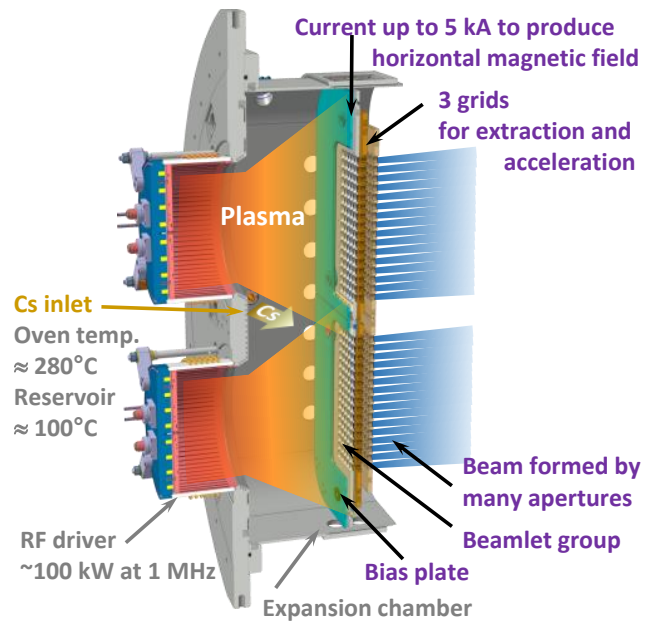

Fig. 1. Vertical cut view through the rear drivers of the ELISE ion source with the basic components labelled.

Each driver houses one gas inlet for either hydrogen or deuterium. At ELISE caesium is evaporated by two Cs ovens through inlets on the lateral side walls (indicated in Fig. 1), whereas at the ITER sources three inlets are foreseen at the backplate of the source. The Cs evaporation is controlled via the temperature of the $\mathrm{Cs}$ reservoir inside the ovens (typically $100^{\circ} \mathrm{C}$ ) whereas all other parts of the oven are kept at much higher temperature to avoid cold spots and hence Cs condensation. Typical evaporation rates are around $2 \mathrm{mg} / \mathrm{h} /$ oven, depending also on the isotope, i.e. a higher evaporation rate is required for deuterium operation (see Sec. 3.2.). The source vessel is kept at elevated temperatures (between $35^{\circ} \mathrm{C}$ and $50^{\circ} \mathrm{C}$ ) and the plasmafacing grid, the plasma grid (PG), is operated between $150^{\circ} \mathrm{C}$ and $200^{\circ} \mathrm{C}$ [6]. Caesium covers the $\mathrm{PG}$ and thus reduces the work function of the molybdenum surface needed for an effective surface conversion of (mostly) hydrogen atoms and positive ions into negative ions.

In negative ion sources for NBI a magnetic filter field of several $\mathrm{mT}$ is needed to reduce the electron temperature and electron density and thus the destruction of negative ions by collisions with energetic electrons (electron stripping). In addition, the amount of co-extracted electrons can be controlled with the magnetic filter field strength and topology with the drawback to reduce the extracted negative ions if the field strength is too strong $[10,11]$. The horizontal magnetic field is created by a current flowing vertically through the PG, indicated in Fig. 1. At ELISE three return conductors are placed beside and between the drivers and up to $5 \mathrm{kA}$ are available creating about $1 \mathrm{mT} / \mathrm{kA}$ in front of the PG. The interplay of the magnetic field with plasma gradients (density, temperature and potentials) results in vertical plasma drifts as seen in the experiments and confirmed by fluid modelling [12]. In the present configuration, the PG current is fed from the bottom, creating a horizontal field in left-right direction (plasma view on the grid system) and an effective plasma drift in upward direction. On the beam side, the field causes a vertical deflection of the beam, in the opposite direction with respect to the plasma drift. For the ITER sources the arrangement of the return conductors is different such that the topology of the field in the source has been optimized and beam deflection downstream the PG is minimized [13] (see also Sec. 3.4).

The ELISE source is well equipped with diagnostics to measure and monitor the plasma parameters: (i) Langmuir probes for the plasma density, electron temperature, plasma potential, (ii) Cavity ringdown spectroscopy (CRDS) for negative ion densities, (iii) tunable diode laser absorption spectroscopy for neutral Cs densities in vacuum and plasma phases, (iv) emission spectroscopy for plasma parameters and Cs monitoring (see for example [8] and references therein). Focus is given to the plasma region close to the grid system $(\approx 2$ $\mathrm{cm}$ from $\mathrm{PG}$ ) to improve the physics understanding of the complex interplay of those parameters in the plasma region where correlations with the beam parameters can be expected.

\subsection{Extraction and acceleration}

The source is at negative potential and negative ions are extracted and accelerated towards the grounded grid (GG), the third grid of the two-stage extraction system. The PG is positively biased against the source walls which are extended by the bias plate surrounding the PG pattern (Fig. 1). This, like the magnetic filter field provides an additional, but not independent, knob to reduce the amount of co-extracted electrons. By controlling the bias current ( $\mathrm{I}_{\text {bias }}$, several Ampere) instead of the bias voltage $\left(\mathrm{U}_{\text {bias, }}\right.$ several $\left.10 \mathrm{~V}\right)$, a more reliable control is achieved, such that this mode is preferred. Negatively charged particles are extracted from the source by extraction voltages $\left(\mathrm{U}_{\text {ex }}\right)$ of up to $10 \mathrm{kV}$ applied between PG and EG. The co-extracted electrons are dumped onto the EG by the deflection field created by permanent magnets in the EG. A total acceleration over both stages of up to $60 \mathrm{kV}$ is possible. Due to limitations in the available high voltage (HV) power supply, extraction and acceleration is only possible in pulsed mode (one extraction phase, called blip, about $10 \mathrm{~s}$ each every $\approx 150 \mathrm{~s}$ ) though the grids and the source are designed for continuous operation. Hence, long pulse operation at ELISE means the source is operating continuously whereas the beam is extracted in blips. The electrical currents flowing onto the grids as well as the current flowing back to the $\mathrm{HV}$ power supply are measured individually. The co-extracted electron current $\left(\mathrm{I}_{\mathrm{e}}\right)$ is given by the current measured on the EG, but may include also secondary or stripped electrons as well as negative ions scraped at the apertures. The total extracted negative ion current $\left(\mathrm{I}_{\mathrm{ex}}\right)$ is assigned to the current flowing back from the ground potential (see, e.g., Refs. $[5,8]$ for a discussion). The current measured on the GG is assumed 
to be caused by stripped and secondary electrons; negative ions with high divergence can contribute as well. The accelerated current (negative ions and neutralized particles) is measured by the diagnostic calorimeter installed $3.5 \mathrm{~m}$ downstream of the grid system [14].

ELISE uses the same grid pattern as the ITER beam sources, having one row with 4 beamlet groups arranged in each of the two grid segments (indicated in Figure 1; a picture is given in Ref. [8]). One beamlet group consists of $16 \times 5$ apertures with $14 \mathrm{~mm}$ diameter each. The total extraction area is $985 \mathrm{~cm}^{2}$. In contrast to the ITER sources, the two beam segments of the EG and those of the GG are insulated against each other, such that the measured currents can be assigned to the top and the bottom segments, offering detection of possible asymmetries. An interlock system stops beam extraction in case of unacceptable heat loads on the EG. Although the segments of the EG are designed to withstand a power load of $200 \mathrm{~kW} /$ segment each, the interlock is set to $125 \mathrm{~kW} /$ segment to account for potential nonuniformities within a grid segment [6]. On the ITER sources, the total value for the power load on the EG is $600 \mathrm{~kW}$; an individual control for the four grid segments of $150 \mathrm{~kW} /$ segment) is not foreseen.

\subsection{Beam}

As indicted in Fig. 1 the large beam is formed by the overlap of 640 individual beamlets. Unlike the ITER NBI grids, all grids in ELISE are flat and not inclined, and beamlet steering is also not provided. The beam is deflected downwards by the downstream part of the magnetic field created by the PG current. The beam shift is in the range of some centimeters at the diagnostic calorimeter. The vertical electron deflection field, created by permanent magnets in the EG, alters in its direction from beamlet row to beamlet row resulting in a horizontal "zig-zag pattern" for which no compensation is integrated at ELISE (but at the ITER sources [15]).

The divergence of a beamlet is linked to its perveance which is defined by the Child-Langmuir law resulting for the first gap (between PG and EG) in Perv. $=\mathrm{I}_{\mathrm{ex}} / \mathrm{U}_{\mathrm{ex}}{ }^{1.5}$. Hence for a fixed extraction voltage the optimum perveance, i.e. the minimum divergence, depends on the extracted ion current. Taking into account additionally the second electrostatic lens formed between the EG and the $\mathrm{GG}$, the divergence for a defined perveance depends also on the voltage ratio for the two gaps, $\mathrm{U}_{\mathrm{acc}} / \mathrm{U}_{\mathrm{ex}}$. The optimum ratio of these voltages is found to be between 5 and 6 , both in experiments and calculations [16]. It is assumed that the beamlets are space-charge compensated within a few centimeters behind the grid system such that the divergence increases no longer. The geometrical arrangement of the grid system for a single aperture of ELISE is shown in Fig. 2. Calculations of the respective beam optics are performed using the IBSimu code [17]. To mimic the non-zero perpendicular energy of the particles entering the meniscus in the experiment a perpendicular temperature is used for the simulation particles, which adds $\sqrt{T_{\perp} / U_{\text {total }}}$ to the divergence [18]. The numerical value of $1 \mathrm{eV}$ is chosen as it represents an upper estimate for the average energy of the negative ions.
The calculations predict a minimum divergence of $11 \mathrm{mrad}$, which is above the $7 \mathrm{mrad}$ calculated for the ITER system. It should be noted that these numbers refer to the global minimum and depends beside the voltages also on the current density. For the ITER targeted current densities at the extraction voltage of $10 \mathrm{kV}$ and maximum available acceleration voltage of $50 \mathrm{kV}$ the predicted divergence is higher, namely $19.5 \mathrm{mrad}$ and $14.4 \mathrm{mrad}$ in the hydrogen and deuterium case, respectively.

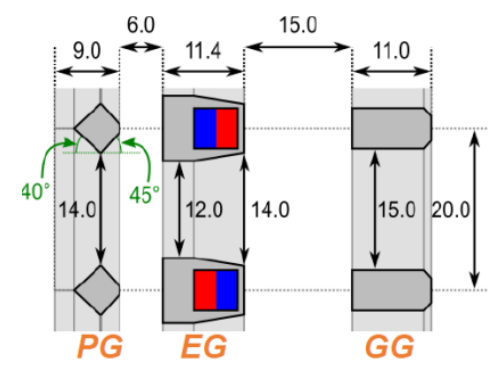

Fig. 2. Details of the grid system at ELISE for a single aperture, showing the dimensions of the apertures of the plasma grid (PG), the extraction grid (EG) and the grounded grid (GG).

Several beam diagnostics are available for monitoring the shape of the beam (tungsten-wire calorimeter), for determining the line-of-sight averaged divergence and intensity (beam emission spectroscopy, BES) and for determination of the beam power with some spatial resolution (diagnostic calorimeter) [19]). The latter allows determining the beam uniformity in terms of symmetry or asymmetry of the two beam segments, or down to a level of the beamlet groups. The 16 vertical lines-of-sight of BES provide a better vertical resolution, however averaged along the observation volume. Details of the positions and capabilities of the different beam diagnostics together with some recent results are given in Ref. [16]. It should be noted that due to beam inhomogeneities and the zig-zag pattern of the negative ions, the line-of-sight averaged divergences resulting from the overlap of several beamlets are typically higher than the one predicted for a single beamlet (see discussion in Sec. 3.3.).

\section{Achievements and challenges}

The progress in the ion source performance is reported and illustrated in Ref. [20] covering the time period from start of operation until mid-2018. In brief, focus was laid first on achieving the target parameters in short pulses ( $20 \mathrm{~s}$ plasma on-time with one extraction blip) for hydrogen while getting also some experience in deuterium operation as well as in operation of long plasma pulses with several extraction blips. Several technical issues had to be solved for reliable operation at high RF powers, i.e. higher than about $60 \mathrm{~kW} /$ driver. Among them were breakdowns in the driver region, cooling problems with the electromagnetic RF-shields and matching of the RF circuit with the tube-based oscillators $[9,20]$. In high power operation and short pulses above $90 \%$ of the target for extracted current densities were achieved for both isotopes, limited in hydrogen only by the maximum 
extracted current which can be handled by the HV power supply and in deuterium by the amount of co-extracted electrons. Focus was then shifted to long pulses, with operation and source conditioning typically in $400 \mathrm{~s}$ pulses hosting three extraction blips. The steepness of the increase of the co-extracted electron current from extraction blip to extraction blip turned out to be a good indicator for a successful run with $1000 \mathrm{~s}$ in hydrogen or 1 hour in deuterium.

\subsection{Long pulses}

The major challenge in long pulse operation ( $>400 \mathrm{~s})$ is to keep the heat load of the co-extracted electrons at the two grid segments of the EG below the interlock value. An asymmetry of up to factors of two is seen for the two segment, its value changing during long pulses, $[6,8,9,20]$. The amount of co-extracted electrons is very sensitive on the Cs conditioning of the source. In an ionion plasma, i.e. a plasma dominated by negative and positive ions, the electrons are the minority and small variations of the work function of the Cs layer at the PG and thus the amount of negative ions in the plasma result in large variations of the electron density. In contrast, variations of the extracted ion current densities are typically below $10 \%$. Hence, the source performance is limited by the co-extracted electrons as RF power and extraction voltage have to be reduced accordingly. Other control parameters are the magnetic field strength and topology together with the bias current which both can also lead (if too strong) to a reduction of the extracted ion current.

The very first demonstration of stable extracted hydrogen ion current density over $90 \%$ of the target for the $1000 \mathrm{~s}$ (probed by six extraction blips) at a global beam symmetry of better than $90 \%$ was achieved in mid2018 [20,21] and represents a milestone for the success of the ITER NBI. The RF power was at the maximum value available, i.e. $75 \mathrm{~kW} /$ driver were applied, and the extracted current of $30 \mathrm{~A}$ was at the limit for the HV power supply. Without those constrains, the ITER target of 33 A could have been reached, as the co-extracted electron-to-ion ratio was below 0.7 for the last extraction blip. The magnetic field strength close to the grid was set to $2 \mathrm{mT}$. The accelerated ion current exceeded the ITER value because the stripping losses in a 3-grid system are lower (measured values are below $10 \%$ ) than for the 7grid system of ITER NBI. Prerequisite for this performance were: (i) the introduction of external permanent magnets at the lateral walls close to the grid system which changes the magnetic field topology at the side of the source, (ii) the installation of potential rods to influence the potentials close to the grid system, and (iii) a dedicated caesium conditioning technique, the so-called "Cs over-conditioning" which also allowed to achieve good reproducibility of this performance without requiring lengthy re-conditioning phases between the pulses. In this setup, a sequence of several $1000 \mathrm{~s}$ pulses at this parameters could be demonstrated as well [20,21]. Pulses at a filling pressure of $0.4 \mathrm{~Pa}$ instead of $0.3 \mathrm{~Pa}$ result in a strong reduction of the co-extracted electrons combined with a slight increase (about $5 \%$ ) of the ion current density. This option should be kept in mind if the stripping losses at the ITER grid system are measured to be lower than the predicted value of $30 \%$.

One of the issues in long pulse operation is providing sufficient caesium flux to the PG surface to maintain the work function at the PG [22,23]. The Cs evaporation rate is a compromise between sufficient deposition of Cs reservoirs at the surface in the vacuum phase and a minimum leakage through the grid system causing breakdowns during extraction. Caesium is redistributed by the plasma and the majority of Cs particles are ionized in the plasma phase. As a positive bias is applied to the $\mathrm{PG}$, Cs ions might be hindered (depending on their energy) to reach the PG. During extraction, positive hydrogen ions created in the grid system by collisions with the background gas are back-accelerated into the source and sputter caesium accumulated at the backplate of the source resulting in an increased Cs density and a reduction of the co-extracted electron current during the extraction blip [23]. With the Cs over-conditioning procedure the Cs amount is higher than the optimum for short pulses resulting in a reduction of negative ion current densities for the first extraction blip preceding a stable $1000 \mathrm{~s}$ phase [21].

\subsection{Deuterium}

Deuterium operation is characterized by a higher amount and dynamics of co-extracted electrons and higher Cs consumption than for hydrogen operation $[8,6,24]$. Hence, a higher magnetic filter field strength of about $4 \mathrm{mT}$ in front of the PG is mandatory for deuterium, whereas for hydrogen the optimum value is between $1.5 \mathrm{mT}$ and $3 \mathrm{mT}$. Additionally, the bias is increased, both (filter field and bias) reducing the extractable negative ion current. The recipe of the Cs over-conditioning which worked well in hydrogen could not be applied to deuterium as the increase of the Cs amount is hindered by the HV breakdowns caused by too much caesium. As a consequence of the poor control of the co-extracted electrons in deuterium, the source is typically operated at reduced RF power and reduced extraction voltage.

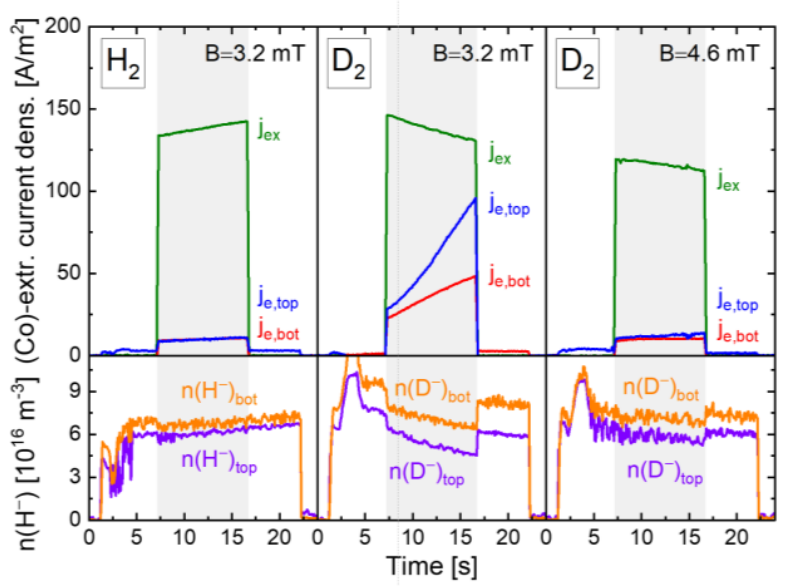

Fig. 3. Extracted current densities (top) and negative ion densities (bottom) measured in about $2 \mathrm{~cm}$ distance to the PG for the transition from hydrogen to deuterium at same magnetic filter field (first and second column) and with adjusted filter field strength for deuterium (third 
column). All data are taken at a filling pressure of $0.3 \mathrm{~Pa}$ and an RF power of $40 \mathrm{~kW} /$ driver

The top part of Fig. 3. gives time traces of extracted current densities from two consecutive short pulses changing from hydrogen to deuterium at same source parameters. The co-extracted electron current densities for the two segments (top and bottom segment) are stable and symmetric for hydrogen whereas a strong spatial and temporal dynamic is evident in deuterium. This can be recovered by increasing the magnetic field strength (third time trace) but with the caveat that the negative ion current density decreases also, but to a much less extent. The slight temporal variations of the extracted ion current are in agreement with the negative ion density measured in front of the PG by the CRDS diagnostics using two lines-of-sight, located in the top and bottom part of the PG (bottom part of Fig. 3.). An asymmetry is also reflected in the negative ion density, being reversed to the one observed for the co-extracted electrons. The overview of the whole sequence is described in Ref. [24] from which the increase of a factor of five in the total co-extracted electron density and a factor of 3-4 higher neutral Cs density is measured in deuterium at same parameters.

The respective pictures of the IR diagnostics at the calorimeter (Fig. 4.) reflect a more intense and better focused beam in the top half and a more diffuse beam in the lower half, giving almost the same picture for deuterium when the magnetic field strength is adapted but at lower total beam power (right picture of Fig. 4 compared to left picture). Comparing hydrogen and deuterium at same magnetic field strength and beam power (left and middle picture of Fig. 4), the beam indicates a better divergence for deuterium, which is confirmed by a detailed analysis. ELISE operates typically in the underperveant branch of the perveance curve (to be seen in Fig. 5) and the observed result reflects the interplay of magnetic field and perveance by changing the current density and the mass of the species.

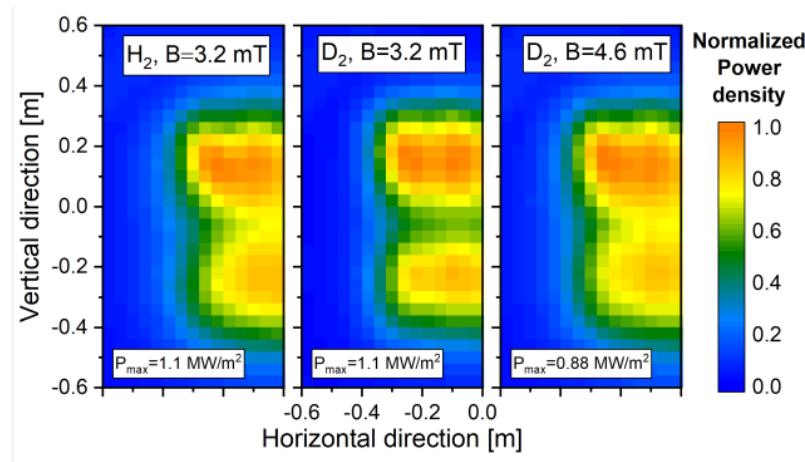

Fig. 4. Left halves of the IR diagnostic at the calorimeter for the same discharges as Fig. 3.

For long pulses, almost $200 \mathrm{~A} / \mathrm{m}^{2}$ have been extracted in deuterium, lasting $2700 \mathrm{~s}$ interrupted only by a faulty temperature control unit [25]. The increase of the ion current compared to the very first one hour pulse, which gave $57 \mathrm{~A} / \mathrm{m}^{2}$ [11], was possible only by the external magnets and the potential rods (as mentioned before in the case of hydrogen) reducing and stabilizing the amount of co-extracted electrons such that the RF power could be increased from $20 \mathrm{~kW} /$ driver to $60 \mathrm{~kW} /$ driver and the extraction voltage from $4.5 \mathrm{kV}$ to $10.5 \mathrm{kV}$. In addition, a two times higher Cs evaporation rate has been applied.

\subsection{Beam divergence}

The beam divergence is derived from the half $1 / \mathrm{e}$ width of the Gaussian fit to the Doppler-shifted peak seen by the BES system, with the assignment of an error bar an error bar of about $10 \%$ which is determined mainly by the statistical error on the raw signal [26].

Figure 5 shows the dependence of the divergence on the acceleration voltage at fixed extraction voltage of $5 \mathrm{kV}$ and an extracted current density of $142 \mathrm{~A} / \mathrm{m}^{2}$ (hydrogen) obtained from a vertical and a horizontal lineof-sight (LoS). Both show a broad minimum around a voltage ratio of about six, but the horizontal LoS reveals with $1.8^{\circ}(31 \mathrm{mrad})$ a higher divergence as the vertical $\operatorname{LoS}\left(1.3^{\circ}, 22 \mathrm{mrad}\right)$. This is attributed mainly to the above mentioned zig-zag deflection due to the fact that the BES detects not only one beamlet row but also signals from rows above and below. Horizontal deflections are seen by the BES at different Doppler-shifted positions very close to each other, thus resulting in a unique and large Doppler peak. Dedicated measurements on the effect and the suppression of the zig-zag deflection carried out at the NITS test facility (QST, Japan) using a CFC title demonstrated that the deflection can cause a horizontal shift spatially equivalent to about one to two beamlets. [27].

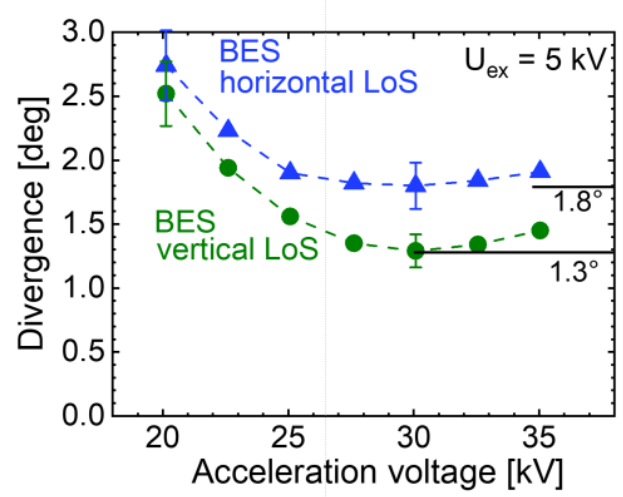

Fig. 5. Divergence measured at ELISE by BES using a vertical and an horizontal line-of-sight. At a filling pressure of $0.3 \mathrm{~Pa}$ the acceleration voltage varies at an extraction voltage of $5 \mathrm{kV}$ and an extracted current density of $143 \mathrm{~A} / \mathrm{m}^{2}$.

Consequently, a comparison of the BES measured divergence of a group of beamlets to the one of a single beamlet has been carried out recently at the test facility BATMAN Upgrade (BUG) [28]. The aperture arrangement is identical to the one at ELISE (Fig. 2.) except that a fourth grid, the repeller grid, is added after the GG being set to the same potential for this dedicated campaign. Figure 6 (a) shows the beamlet group $(14 \times 5$ apertures) installed at BUG (due to space constraints, it is reduced by two rows compared to ELISE) in which the upper half of the beamlet group of the PG is masked except a central aperture. The lower half is kept open such that the beam from a single beamlet and the one from the group of beamlets (Fig. 6(a)) can be diagnosed 
simultaneously. BES is installed at $1.3 \mathrm{~m}$ behind the GG using lines-of- sight representing the signal of the single beamlet and the one from the beamlet group. In addition, a CFC tile (mini-STRIKE [29]) has been installed in collaboration with Consorzio RFX, Padua, to allow for a comparison of the results for the single beamlet [28].

A similar parameter variation as for ELISE has been carried out, such that Fig. 5. and Fig. 6(b) can be compared directly. The horizontal LoS from BES measuring the group of beamlets yield the same behavior, a minimum of the divergence at $1.8^{\circ}$ although at BUG only one beamlet group is measured compared to the four at ELISE. Part (b) of Fig. 6 shows also that the measurements of the single beamlet results in a much lower divergence with the minimum shifted to slightly higher acceleration voltages. In addition, an excellent agreement between BES and the mini-STRIKE analysis is observed, demonstrating also the reliability of the BES system. The perveance minimum is with $0.8^{\circ}-1^{\circ}$ close to the values predicted by the beamlet simulations. More systematic investigations on the beam divergence of a single beamlet in comparison with calculations and with the divergence of a group of beamlets are foreseen at BUG. For this purpose a new CFC tile $\left(38 \times 14 \mathrm{~cm}^{2}\right)$ at a distance of $0.8 \mathrm{~m}$ from the GG will be installed.

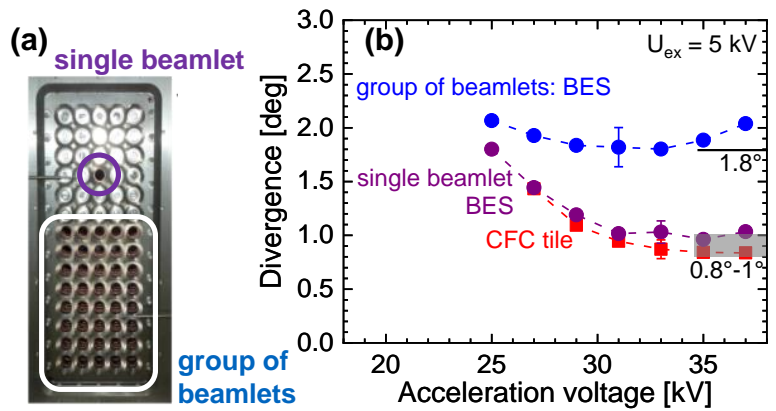

Fig. 6. (a) View on the beamlet group at BATMAN Upgrade with the modified configuration for measuring the divergence of a single beamlet and that of a group of beamlet simultaneously. (b) Results from BES and the CFC tile (mini-STRIKE) for a variation of the acceleration voltage at fixed extraction voltage of $5 \mathrm{kV}$ and an extracted current density of $130 \mathrm{~A} / \mathrm{m}^{2}$ at a pressure of $0.3 \mathrm{~Pa}$.

It should be pointed out that the grid system of BUG and ELISE is prone to beamlet scraping at the EG at low extraction voltages (approx. less than $6 \mathrm{kV}$ ) and high current density [28].

\subsection{MITICA-like filter field}

The magnetic filter field of the ITER NBI source differs in some aspects from the field at ELISE and will be tested at MITICA (part of the NBTF) representing the prototype of the ITER beamline. The magnetic filter field is generated by the current through the PG (as at ELISE) with the boundary condition to have a fixed value of the magnetic field in front of the grid of $3 \mathrm{mT}$ and a weak long-range field of $0.5 \mathrm{mT}$ (at the exit of the accelerator) to deflect secondary and stripped electrons out of the beam [14]. For that purpose additional side conductors are placed beside the source in the PG plane and two remote return conductors are positioned about $1 \mathrm{~m}$ behind the source. The current of side conductors is flowing back through the three return conductors beside the drivers. The PG current is returned through the remote return conductors. The desired field strengths are given by having a fixed value of $17 \%$ of the total current for the side conductors, namely $0.7 \mathrm{kA}$ out of $4.2 \mathrm{kA}$. As a secondary effect, the magnetic field in the driver is higher than for ELISE which might cause problems with plasma sustainment in the driver. In order to verify the source performance at an early stage, this field configuration has been mimicked at ELISE [20] (Fig. 6 of [20] gives a comparison of the 2D-magnetic field topology of the MITICA-like and the standard ELISE configuration).

Figure 7 shows the extracted current densities for $300 \mathrm{~s}$ pulses with three extraction blips in hydrogen for both magnetic field configurations at similar field strengths in front of the grid. In the MITICA-like configuration the ion current density is slightly higher and, even more important, the co-extracted electron current density is reduced by a factor of two. In both cases the electron-to-ion ratio is well below one or even below 0.5 . The magnetic field is chosen such that the extracted ion current density is not reduced but the electrons sufficiently suppressed. As a general observation, magnetic field strengths lower than the $3 \mathrm{mT}$ are sufficient in the MITICA-like configuration for hydrogen operation and need to be adapted to optimize the interplay of Cs conditioning, bias and field strength on the coextracted electrons. Initial experiments indicate that increasing the current fraction through the side return conductors (i.e. using higher values than the $17 \%$ specified for MITICA) can result in a strongly reduced coextracted electron current (about 30\%) and additionally significantly improve the top-bottom symmetry of the coextracted electrons.

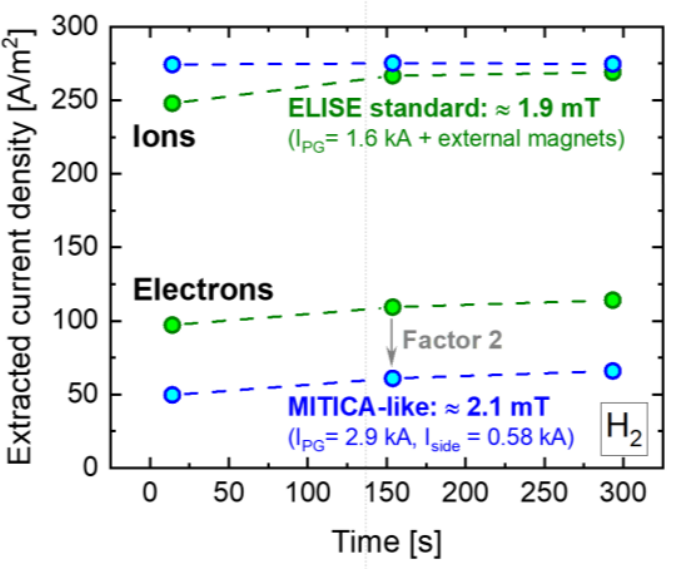

Fig. 7. Extracted current densities (negative ions and coextracted electrons) for the two magnetic field configurations: the MITICA-like and the ELISE standard one [20] at same ion source parameters for a 300 s pulse with 3 extraction blips. Hydrogen operation at a filling pressure of $0.3 \mathrm{~Pa}$.

As already pointed out, higher magnetic field strengths are needed in deuterium operation. Figure 8 shows the respective comparison for the best long pulses. In the ELISE standard configuration, a field of $4.6 \mathrm{mT}$ 
was needed to suppress the co-extracted electrons which are shown separately for the two grid segments. Unfortunately, a false signal stopped the pulse at $45 \mathrm{~min}$. For the MITICA-like field the maximum field strength was limited by the available power supply. Figure 8 shows that the current density of the negative ions is slightly lower, which is attributed to a somewhat different Cs conditioning. Due to the higher Cs dynamics in deuterium plasmas, stable and reproducible Cs conditions are difficult to achieve. As Figure 8 illustrates, the increase of the co-extracted electrons with time is much steeper in the MITICA-like configuration than in the ELISE standard case. This pulse has been terminated by the interlock for the power load on the grid segments of the EG. The same asymmetry of the co-extracted electron current densities is seen for the two grid segments; for the top segment a factor of two higher values are obtained. In principle, the asymmetry can be influenced by the current through the side conductors (not shown here), i.e. increasing this current would have resulted in a lower asymmetry. In this case this was unfortunately not possible due to the limitations by the power supply.

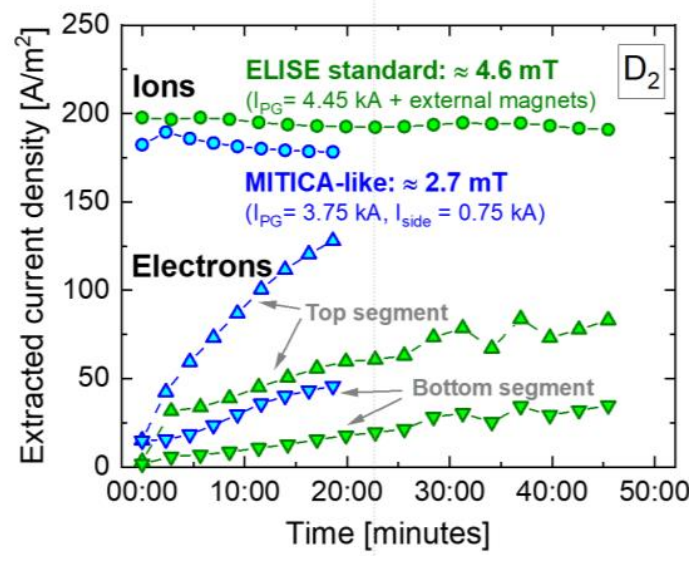

Fig. 8. Extracted current densities (negative ions and coextracted electrons for the two grid segments separately) for the two magnetic field configurations: the MITICAlike and the ELISE standard one [20] at same ion source parameters for pulses up to $45 \mathrm{~min}$. Deuterium operation at a filling pressure of $0.3 \mathrm{~Pa}$.

The vertical non-uniformity of the co-extracted electrons demonstrate clearly the need to have the interlock for grid protection at least separated for the individual grid segments, in particular in view of the ITER source being twice the height of the ELISE source. The tests indicated also that the envisaged $3 \mathrm{mT}$ are not sufficient for deuterium and that flexible currents for the two circuits would be very advisable for optimization of the source performance.

\section{Conclusion}

The ELISE test facility gave already valuable input to the design, commissioning and operation of the ITER-size NBI ion sources, among them the technological aspects as the confirmation of the grid manufacturing and the solution for the encountered RF issues to achieve reliable long pulse operation at high power. Besides avoiding RF breakdowns and providing sufficient cooling of components close to the RF coupling, the replacement of the currently planned tube-based oscillators by solid-state $\mathrm{RF}$ generators is highly advisable.

Among the challenges in achieving the ITER requirements in terms of extracted current densities at a ratio of co-extracted electrons to ions below one for long pulses are the suppression of the co-extracted electrons by the complex interplay of the magnetic field configuration, the bias and the introduced potential rods, together with the Cs conditioning. Scenarios have been developed for which the ITER target have been almost reached for hydrogen in $1000 \mathrm{~s}$ pulses: more than $90 \%$ of the extracted negative ion current (limited at ELISE by the available HV power supply and the available RF power). The demonstration of repetitive pulses at this high performance represents a milestone for the ITER HNB operation at its first phase. For deuterium the performance is limited by the much larger amount of co-extracted electrons; so far $65 \%$ percent of the required extracted current density has been demonstrated for long pulses. As the dedicated campaign for the isotope change clearly demonstrated an increase of the co-extracted electrons by a factor of up to five at same source parameters, this has to be counteracted by an increase of the magnetic filter field strength which reduces also the extracted negative ion current but to a minor extent. The filter field topology to be used at the prototype injector MITICA and at the ITER HNB was mimicked at ELISE and showed a good performance. However, it is advisable to keep the magnetic filter field strength as well as the currents flowing to the remote return conductors and side conductors flexible to adjust for the isotope and for the fine-tuning needed during Cs conditioning of the ion source. In addition, a higher Cs dynamics is evident in deuterium with the need of increased caesium evaporation and control. Regarding the co-extracted electrons, the asymmetry of the power deposited into the two grid segments makes measurements of the heat load for the individual grid segments for the ITER-size sources desirable such that individual interlocks can be applied to protect damages of the EG.

Regarding the beam homogeneity the global uniformity of better than $90 \%$ is usually achieved, for both hydrogen and deuterium, however the present diagnostic does not allow to go down to the precise measurements of homogeneity within a beamlet group or even among single beamlets. Investigations on the beam divergence carried out so far at ELISE and at the accompanying test facility BATMAN Upgrade revealed a higher divergence for the horizontal LoS of the BES measurements than for the vertical ones. As the zig-zag deflection of the beamlets in horizontal direction will be compensated, this should be no issue for the ITER-size prototype sources at the NBTF. On the other hand, the beam optic investigations indicated that additional effects contribute to the global beam divergence than captured with the present simulations for one beamlet. The ITER-size source at the SPIDER test facility (part of the NBTF) will have dedicated diagnostics accompanied by modelling efforts such that this topic can be well addressed. Such 
campaigns will be accompanied by further studies at the BATMAN Upgrade test facility.

As a next step, the constraint of probing the beam properties at the ELISE test facility by extraction blips will become obsolete as the present available HV power supply will be replaced by a steady-state power supply in near future. Consequences on the Cs dynamics and in particular the co-extracted electrons are expected and will give also valuable input for the operation of the ITER-size ion sources.

\section{References}

[1] R. Hemsworth et al., Nucl. Fusion 49 (2009) 045006.

[2] R. Hemsworth et al., New J. Phys. 19 (2017) 025005.

[3] R. Nocentini et al., IEEE Transactions on Plasma Science 42 (2014) 616.

[4] A. Masiello et al., Fusion Eng. Des. 86 (2011) 860.

[5] E. Speth et al., Nucl. Fusion 46 (2006) S220-S238.

[6] U. Fantz, et al., Nucl. Fusion 57 (2017) 116007.

[7] V. Toigo et al., Nucl. Fusion 59 (2019) 086058.

[8] B. Heinemann et al., New J. Phys. 19 (2017) 015001.

[9] B. Heinemann et al., Fusion Eng. Des. 136 (2018) 569.

[10] P. Franzen et al., Plasma Phys. Control. Fusion 53 (2011) 115006.

[11] D. Wünderlich et al., Plasma Phys. Control. Fusion 58 (2016) 125005.

[12] U. Fantz et al., Plasma Phys. Control. Fusion 53 (2011) 115006.

[13] G. Chitarin et al., AIP Conf. Proc. 1515 (2013) 217.

[14] R. Nocentini et al., Fusion Eng. Des. 109-111 (2016) 673.

[15] G. Chitarin et al., AIP Conference Proceedings 1655 (2015) 040008.

[16] F. Bonomo et al., On the Vertical Uniformity of an ITERlike Large Beam, contribution to the ISFNT conference 2019, submitted to Fusion Eng. Des.

[17] T. Kalvas et al., Rev. Sci. Instrum. 81 (2010) $02 B 703$.

[18] J. Kim et al., J. Appl. Phys. 49 (1978) 517.

[19] R. Nocentini et al., Fusion Eng. Des. 88 (2013) 913.

[20] B. Heinemann et al., Fusion Eng. Des. 146 (2019) 455.

[21] D. Wünderlich et al., Nucl. Fusion 59 (2019) 084001.

[22] U. Fantz et al., Fusion Eng. Des. 136 (2018) 340.

[23] A. Mimo et al., AIP Conference Proceedings 2052 (2018) 040009 .

[24] D. Wünderlich et al., Rev. Sci. Instrum. 90 (2019) 113304.

[25] W. Kraus et al., Rev. Sci. Instrum. 89 (2018) 052102.

[26] B. Zaniol, J. Quant. Spectrosc. Radiat. Transf. 112 (2011) 513.

[27] G. Chitarin et al., Fusion Eng. Des. 146 (2019) 792.

[28] C. Wimmer et al., Rev. Sci. Instrum. 91 (2020) 013509.

[29] G. Serianni et al., Rev. Sci. Instrum. 85 (2014) 02 A736. 